\title{
Note on theoretical and practical solvability of a class of discrete equations generalizing the hyperbolic-cotangent class
}

\author{
Stevo Stević, ${ }^{1,2^{*}}$, Bratislav Iričanin ${ }^{3,4}$, Witold Kosmala ${ }^{5}$ and Zdeněk Šmarda ${ }^{6}$
}

"Correspondence: sstevic@ptt.rs
${ }^{1}$ Mathematical Institute of the
Serbian Academy of Sciences, Knez
Mihailova 36/III, 11000 Beograd,
Serbia
2Department of Medical Research,
China Medical University Hospital,
China Medical University, Taichung
40402 , Taiwan, Republic of China
Full list of author information is
available at the end of the article

\section{Springer}

\begin{abstract}
There has been some recent interest in investigating the hyperbolic-cotangent types of difference equations and systems of difference equations. Among other things their solvability has been studied. We show that there is a class of theoretically solvable difference equations generalizing the hyperbolic-cotangent one. Our analysis shows a bit unexpected fact, namely that the solvability of the class is based on some algebraic relations, not closely related to some trigonometric ones, which enable us to solve them in an elegant way. Some examples of the difference equations belonging to the class which are practically solvable are presented, as well as some interesting comments on connections of the equations with some iteration processes.
\end{abstract}

MSC: 39A45

Keywords: Difference equation; Theoretical solvability; Practical solvability; Equations solvable in a closed form

\section{Introduction}

As usual, by $\mathbb{N}, \mathbb{N}_{0}, \mathbb{Z}, \mathbb{R}$, and $\mathbb{C}$ we denote the sets of natural numbers, nonnegative integers, integers, real numbers, and complex numbers, respectively, whereas the notation $l=\overline{s, t}$, when $s, t \in \mathbb{Z}$ and $s \leq t$, is used instead of writing $s \leq l \leq t, l \in \mathbb{Z}$.

Now we present some motivations for the investigation in this paper and several interesting connections among some classes of difference equations and iteration processes.

\subsection{A quick overview of some old results on solvability}

After discovering some solvable classes of linear difference equations and presenting a few basic methods for solving them (see, for example, $[4,6,7,9,15,16]$ ), some researchers started investigating solvability of some classes of nonlinear difference equations and systems of difference equations.

An important paper in this direction, as well as in solvability theory in whole, is [17] by Laplace, where he, among several other ones, investigated the solvability of the difference

(c) The Author(s) 2021. This article is licensed under a Creative Commons Attribution 4.0 International License, which permits use sharing, adaptation, distribution and reproduction in any medium or format, as long as you give appropriate credit to the original author(s) and the source, provide a link to the Creative Commons licence, and indicate if changes were made. The images or other third party material in this article are included in the article's Creative Commons licence, unless indicated otherwise in a credit line to the material. If material is not included in the article's Creative Commons licence and your intended use is not permitted by statutory regulation or exceeds the permitted use, you will need to obtain permission directly from the copyright holder. To view a copy of this licence, visit http://creativecommons.org/licenses/by/4.0/. 
equation

$$
x_{n+1}=x_{n}^{2}-2, \quad n \in \mathbb{N}_{0} .
$$

It should be mentioned that the method for showing the solvability of the equation used by Laplace is based on some simple algebraic relations. He presented the initial value $x_{0}$ in the form

$$
x_{0}=\alpha+\frac{1}{\alpha} \text {, }
$$

and by calculating first few members of $x_{n}$ found a general solution to the equation in terms of $\alpha$ and $n$.

It is interesting to note that by employing the change of variables

$$
x_{n}=2 \widehat{x}_{n}, \quad n \in \mathbb{N}_{0},
$$

equation (1) becomes

$$
\widehat{x}_{n+1}=2 \widehat{x}_{n}^{2}-1, \quad n \in \mathbb{N}_{0},
$$

which resembles the double angle identity for the cosine function. This fact suggests its solvability. Namely, one can expect that a sequence of cosines satisfies the equation. Bearing in mind that

$$
\cos z=\frac{1}{2}\left(e^{i z}+\frac{1}{e^{i z}}\right)
$$

it becomes clear why the use of the quantity $\alpha+\frac{1}{\alpha}$ enabled Laplace to solve equation (1).

Laplace did not give the explanation, but it was realized by researchers of the time that difference equations which have forms to some trigonometric formulas could be solvable.

Later books, besides solvability of some classes of linear difference equations, also mention some of solvable nonlinear ones (see, e.g., [5, 10, 12, 14, 18-21]), but to a small extent.

\subsection{Solvability of some equations and iteration processes}

Difference equations naturally appear in many areas of science, among other ones, in numerical mathematics for iteration processes serving for calculating some quantities such as roots of some functions (see, e.g., $[8,11]$ ). One of root-finding algorithms is the secant method. Recall that if the initial values $x_{0}, x_{1}$ are real, to find a root of a function $f$, one can construct the line through the points $\left(x_{0}, f\left(x_{0}\right)\right)$ and $\left(x_{1}, f\left(x_{1}\right)\right)$ and find the intersection point of the line with the $x$-axis, that is, the root of the linear function

$$
y=f\left(x_{1}\right)+\frac{f\left(x_{1}\right)-f\left(x_{0}\right)}{x_{1}-x_{0}}\left(x-x_{1}\right),
$$

which is equal to

$$
x_{2}=x_{1}-f\left(x_{1}\right) \frac{x_{1}-x_{0}}{f\left(x_{1}\right)-f\left(x_{0}\right)} .
$$


Repeating the procedure for the points $\left(x_{1}, f\left(x_{1}\right)\right)$ and $\left(x_{2}, f\left(x_{2}\right)\right)$, and so on, we obtain the iteration process

$$
\begin{aligned}
x_{n+2} & =x_{n+1}-f\left(x_{n+1}\right) \frac{x_{n+1}-x_{n}}{f\left(x_{n+1}\right)-f\left(x_{n}\right)} \\
& =\frac{f\left(x_{n+1}\right) x_{n}-f\left(x_{n}\right) x_{n+1}}{f\left(x_{n+1}\right)-f\left(x_{n}\right)}, \quad n \in \mathbb{N}_{0},
\end{aligned}
$$

for the secant method.

It is an interesting fact that if in (2) we choose the function

$$
f(x)=x^{2}-a
$$

then after some simple calculations we get

$$
x_{n+2}=\frac{x_{n+1} x_{n}+a}{x_{n+1}+x_{n}}, \quad n \in \mathbb{N}_{0},
$$

which is an iteration process for calculating the square root of number $a$ (it is a root of function (3)).

Another interesting fact is that equation (4) is solvable in a closed form. Moreover, the equation belongs to the class of theoretically solvable difference equations

$$
x_{n+k}=\frac{x_{n+l} x_{n}+a}{x_{n+l}+x_{n}}, \quad n \in \mathbb{N}_{0},
$$

where $k, l \in \mathbb{N}_{0}, l<k$ (see $\left.[26,35]\right)$.

These facts suggest that equation (5) can be also obtained from an iteration process. Indeed, if in the following modification of the secant iteration process (with delayed indices)

$$
\begin{aligned}
x_{n+k} & =x_{n+l}-f\left(x_{n+l}\right) \frac{x_{n+l}-x_{n}}{f\left(x_{n+l}\right)-f\left(x_{n}\right)} \\
& =\frac{f\left(x_{n+l}\right) x_{n}-f\left(x_{n}\right) x_{n+l}}{f\left(x_{n+l}\right)-f\left(x_{n}\right)},
\end{aligned}
$$

for $n \in \mathbb{N}_{0}$, we choose function (3), we really get equation (5). Of course, the process is determined if the initial values $x_{j}, j=\overline{0, k-1}$ are given.

One of the facts that suggest solvability of the difference equations in (5) is that they look like the cotangent sum formula. Note also that when $a \neq 0$ a linear change of variables reduces the equation to the case $a=1$, which exactly looks like the cotangent sum formula. For some generalizations to systems of cotangent-type difference equations, see, for example, recent papers $[30,33]$ and the related references therein.

Some other recent results on solvability and invariants of difference equations and systems and their applications can be found, for example, in [3, 13, 22-25, 27-29, 31$33,35,37]$ and the references therein.

\subsection{Our aim}

Motivated by all the above mentioned, here we consider the following class of difference equations:

$$
x_{n+k}=\frac{x_{n+l} x_{n}-a b}{x_{n+l}+x_{n}-a-b}, \quad n \in \mathbb{N}_{0} \text {, }
$$


where $k \in \mathbb{N}, l \in \mathbb{N}_{0}, l<k, a, b \in \mathbb{C}$ and $x_{j} \in \mathbb{C}, j=\overline{0, k-1}$, which naturally generalizes equation (5).

Our aim is to show that equation (7) is theoretically solvable, and that it is a consequence of some pure algebraic relations which are essentially not closely connected to some trigonometric type relations. This shows that the solvability of equation (5) also relies on the same algebraic relation.

\section{Main results}

This section presents our main results in this paper. We study the solvability of equation (7) by considering several cases separately.

Case $a=b=0$. If $a=b=0$, then equation (7) becomes

$$
x_{n+k}=\frac{x_{n+l} x_{n}}{x_{n+l}+x_{n}}, \quad n \in \mathbb{N}_{0} .
$$

By using the change of variables

$$
x_{n}=\frac{1}{u_{n}}, \quad n \in \mathbb{N}_{0},
$$

equation (8) is transformed to the following one:

$$
u_{n+k}=u_{n+l}+u_{n}, \quad n \in \mathbb{N}_{0},
$$

which is a homogeneous linear difference equation with constant coefficients of $k$ th order.

It is well known that the linear difference equations with constant coefficients are theoretically solvable (see, for example, $[4,5,10,12,18-21]$ ), from which theoretical solvability of equation (8) follows. This fact and the fact that by using the change of variables (9) equation (8) is transformed to equation (10) are well known. For example, in [2, Problem 8.16.9] a special case of equation (8) with $k=2$ is solved in this way. Moreover, such equations frequently appear (see, for example, recent paper [30]). Therefore, the case is of no special interest. Nevertheless, some special cases of equation (8) will be solved in a closed form later in the paper.

From now on we consider equation (7) under the following assumption:

$$
a \neq 0 \quad \text { or } \quad b \neq 0 \text {. }
$$

Case $a+b=0$. If $a+b=0$ and (11) holds, then equation (7) becomes

$$
x_{n+k}=\frac{x_{n+l} x_{n}+a^{2}}{x_{n+l}+x_{n}}, \quad n \in \mathbb{N}_{0}
$$

which is a special case of equation (5). As we have already mentioned, the solvability of equation (5) has been thoroughly investigated (see $[26,35]$ ). Hence, we will not consider this case in the paper. Note also that since (11) holds, from $a+b=0$, we have $a=-b \neq 0$.

Case $a \neq 0$ or $b \neq 0, a \neq b$. In this case equation (7) no more has a form of cotangentsum formula. Since we do not have a typical trigonometric formula hint for suggesting its solvability, another hint should be found. 
First, note that the fixed points of the equation satisfy the algebraic equation

$$
x^{*}=\frac{\left(x^{*}\right)^{2}-a b}{2 x^{*}-a-b},
$$

from which it easily follows that

$$
x_{1}^{*}=a \quad \text { and } \quad x_{2}^{*}=b .
$$

This observation together with the form of equation (7) suggests to consider the following quantities/sequences:

$$
x_{n}-x_{1}^{*} \text { and } x_{n}-x_{2}^{*}
$$

for $n \in \mathbb{N}_{0}$.

From (7) and by some simple calculations, it follows that

$$
x_{n+k}-b=\frac{x_{n+l} x_{n}-b\left(x_{n+l}+x_{n}\right)+b^{2}}{x_{n+l}+x_{n}-a-b}, \quad n \in \mathbb{N}_{0} \text {, }
$$

and

$$
x_{n+k}-a=\frac{x_{n+l} x_{n}-a\left(x_{n+l}+x_{n}\right)+a^{2}}{x_{n+l}+x_{n}-a-b}, \quad n \in \mathbb{N}_{0} .
$$

From (14) and (15) we easily obtain

$$
\frac{x_{n+k}-b}{x_{n+k}-a}=\frac{\left(x_{n+l}-b\right)\left(x_{n}-b\right)}{\left(x_{n+l}-a\right)\left(x_{n}-a\right)}, \quad n \in \mathbb{N}_{0} .
$$

By using the change of variables

$$
z_{n}=\frac{x_{n}-b}{x_{n}-a}, \quad n \in \mathbb{N}_{0},
$$

equation (16) is transformed to

$$
z_{n+k}=z_{n+l} z_{n}, \quad n \in \mathbb{N}_{0} .
$$

It is also known that equation (18) is theoretically solvable. Namely, its solvability is closely related to the solvability of equation (10). Some books on difference equations wrongly suggest taking the logarithm of both sides of the equation and then application of the change of variables

$$
\widehat{y}_{n}=\ln z_{n}, \quad n \in \mathbb{N}_{0} .
$$

This is only justified if all $z_{n}$ are positive numbers. Fortunately, there are some correct procedures for finding closed form formulas for general solution to equation (18). We have use them, for example, in [34] (see also the related references therein). 
Equation (10) is certainly practically solvable if $k \leq 4$. For $k \geq 5$, the characteristic polynomial $\lambda^{k}-\lambda^{l}-1$ associated with the equation can be certainly solved by radicals when $k \leq 4$. However, if $k \geq 5$, then by a known theorem [1], it need not be solvable.

Hence, for $k \leq 4$, closed form formulas for solutions to equation (10), and consequently closed form formulas for solutions to equation (18), can be found. We can use such obtained formulas in the formula

$$
x_{n}=\frac{a z_{n}-b}{z_{n}-1}, \quad n \in \mathbb{N}_{0} \text {, }
$$

which easily follows from (17).

Hence, we can claim that the following special cases of equation (18) are certainly practically solvable: 1) $k=2, l=1$; 2) $k=3, l=1$; 3) $k=3, l=2$; 4) $k=4, l=1$; 5) $k=4, l=2$; 6) $k=4, l=1$. The equation in these cases has been solved in some of our papers (see, e.g., [34]) and the following result holds.

Theorem 1 The following statements hold.

(a) General solution to equation (18) with $k=2$ and $l=1$ is given by the formula

$$
z_{n}=z_{1}^{f_{n}} z_{0}^{f_{n-1}}, \quad n \in \mathbb{N}_{0}
$$

where $f_{n}$ is the Fibonacci sequence (see, e.g., [38]).

(b) General solution to equation (18) with $k=3$ and $l=1$ is given by the formula

$$
z_{n}=z_{2}^{\alpha_{n-3}} z_{1}^{\alpha_{n-2}} z_{0}^{\alpha_{n-4}}, \quad n \in \mathbb{N}_{0}
$$

where the sequence $\alpha_{n}$ is given by

$$
\alpha_{n}=\sum_{j=1}^{3} \frac{t_{j}^{n+3}}{P_{3}^{\prime}\left(t_{j}\right)}, \quad n \in \mathbb{Z}
$$

where $P_{3}(t)=t^{3}-t-1$ and $t_{j}, j=\overline{1,3}$, are its zeros.

(c) General solution to equation (18) with $k=3$ and $l=2$ is given by the formula

$$
z_{n}=z_{2}^{\beta_{n-2}} z_{1}^{\beta_{n-4}} z_{0}^{\beta_{n-3}}
$$

where the sequence $\beta_{n}$ is given by

$$
\beta_{n}=\sum_{j=1}^{3} \frac{t_{j}^{n+2}}{Q_{3}^{\prime}\left(t_{j}\right)}, \quad n \in \mathbb{Z},
$$

where $Q_{3}(t)=t^{3}-t^{2}-1=0$ and $t_{j}, j=\overline{1,3}$, are its zeros.

(d) General solution to equation (18) with $k=4$ and $l=1$ is given by the formula

$$
z_{n}=z_{3}^{\gamma_{n-5}} z_{2}^{\gamma_{n}-4} z_{1}^{\gamma_{n-3}} z_{0}^{\gamma_{n-6}},
$$


where the sequence $\gamma_{n}$ is given by

$$
\gamma_{n}=\sum_{j=1}^{4} \frac{t_{j}^{n+5}}{R_{4}^{\prime}\left(t_{j}\right)}, \quad n \in \mathbb{Z}
$$

where $R_{4}(t)=t^{4}-t-1=0$ and $t_{j}, j=\overline{1,4}$, are its zeros.

(e) General solution to equation (18) with $k=4$ and $l=2$ is given by the formulas

$$
\begin{aligned}
& z_{2 n}=z_{2}^{f_{n}} z_{0}^{f_{n-1}}, \quad n \in \mathbb{N}_{0}, \\
& z_{2 n+1}=z_{3}^{f_{n}} z_{1}^{f_{n-1}}, \quad n \in \mathbb{N}_{0},
\end{aligned}
$$

where $f_{n}$ is the Fibonacci sequence.

(f) General solution to equation (18) with $k=4$ and $l=3$ is given by the formula

$$
z_{n}=z_{3}^{\delta_{n-3}} z_{2}^{\delta_{n-6}} z_{1}^{\delta_{n-5}} z_{0}^{\delta_{n-4}}
$$

where the sequence $\delta_{n}$ is given by

$$
\delta_{n}=\sum_{j=1}^{4} \frac{t_{j}^{n+3}}{S_{4}^{\prime}\left(t_{j}\right)}, \quad n \in \mathbb{Z},
$$

where $S_{4}(t)=t^{4}-t^{3}-1=0$ and $t_{j}, j=\overline{1,4}$, are its zeros.

From Theorem 1 , and by using (20) as well as relation (17) with $n=\overline{0,3}$, we obtain the following result.

Theorem 2 Consider equation (7). Assume that $a, b \in \mathbb{C}, a \neq b$, and $a+b \neq 0$. Then the following statements hold:

(a) Assume that $k=2, l=1$. Then the general solution to equation ( 7 ) is given by

$$
x_{n}=\frac{a\left(\frac{x_{1}-b}{x_{1}-a}\right)^{f_{n}}\left(\frac{x_{0}-b}{x_{0}-a}\right) f_{n-1}-b}{\left(\frac{x_{1}-b}{x_{1}-a}\right) f_{n}\left(\frac{x_{0}-b}{x_{0}-a}\right) f_{n-1}-1}, \quad n \in \mathbb{N}_{0},
$$

where $f_{n}$ is the Fibonacci sequence.

(b) Assume that $k=3, l=1$. Then the general solution to equation (7) is given by

$$
x_{n}=\frac{a\left(\frac{x_{2}-b}{x_{2}-a}\right)^{\alpha_{n-3}}\left(\frac{x_{1}-b}{x_{1}-a}\right)^{\alpha_{n-2}}\left(\frac{x_{0}-b}{x_{0}-a}\right)^{\alpha_{n-4}}-b}{\left(\frac{x_{2}-b}{x_{2}-a}\right)^{\alpha_{n-3}}\left(\frac{x_{1}-b}{x_{1}-a}\right)^{\alpha_{n-2}}\left(\frac{x_{0}-b}{x_{0}-a}\right)^{f_{n-1}}-1}, \quad n \in \mathbb{N}_{0},
$$

where the sequence $\alpha_{n}$ is given by (21).

(c) Assume that $k=3, l=2$. Then the general solution to equation ( 7 ) is given by

$$
x_{n}=\frac{a\left(\frac{x_{2}-b}{x_{2}-a}\right)^{\beta_{n-2}}\left(\frac{x_{1}-b}{x_{1}-a}\right)^{\beta_{n-4}}\left(\frac{x_{0}-b}{x_{0}-a}\right)^{\beta_{n-3}}-b}{\left(\frac{x_{2}-b}{x_{2}-a}\right)^{\beta_{n-2}}\left(\frac{x_{1}-b}{x_{1}-a}\right)^{\beta_{n-4}}\left(\frac{x_{0}-b}{x_{0}-a}\right)^{\beta_{n-3}-1}}, \quad n \in \mathbb{N}_{0},
$$

where the sequence $\beta_{n}$ is given by (22). 
(d) Assume that $k=4, l=1$. Then the general solution to equation (7) is given by

$$
x_{n}=\frac{a\left(\frac{x_{3}-b}{x_{3}-a}\right)^{\gamma_{n-5}}\left(\frac{x_{2}-b}{x_{2}-a}\right)^{\gamma_{n-4}}\left(\frac{x_{1}-b}{x_{1}-a}\right)^{\gamma_{n-3}}\left(\frac{x_{0}-b}{x_{0}-a}\right)^{\gamma_{n-6}-b}}{\left(\frac{x_{3}-a}{x_{3}-a} \gamma_{n-5}\left(\frac{x_{2}-b}{x_{2}-a}\right)^{\gamma_{n-4}}\left(\frac{x_{1}-b}{x_{1}-a}\right)^{\gamma_{n-3}}\left(\frac{x_{0}-b}{x_{0}-a}\right)^{\gamma_{n-6}-1}\right.}, \quad n \in \mathbb{N}_{0},
$$

where the sequence $\gamma_{n}$ is given by (23).

(e) Assume that $k=4, l=2$. Then the general solution to equation (7) is given by

$$
\begin{aligned}
& x_{2 n}=\frac{a\left(\frac{x_{2}-b}{x_{2}-a}\right) f_{n}\left(\frac{x_{0}-b}{x_{0}-a}\right) f_{n-1}-b}{\left(\frac{x_{2}-b}{x_{2}-a}\right) f_{n}\left(\frac{x_{0}-b}{x_{0}-a}\right) f_{n-1}-1}, \quad n \in \mathbb{N}_{0}, \\
& x_{2 n+1}=\frac{a\left(\frac{x_{3}-b}{x_{3}-a}\right) f_{n}\left(\frac{x_{1}-b}{x_{1}-a}\right) f_{n-1}-b}{\left(\frac{x_{3}-b}{x_{3}-a}\right) f_{n}\left(\frac{x_{1}-b}{x_{1}-a}\right)^{f_{n-1}}-1}, \quad n \in \mathbb{N}_{0},
\end{aligned}
$$

where $f_{n}$ is the Fibonacci sequence.

(f) Assume that $k=4, l=3$. Then the general solution to equation (7) is given by

$$
x_{n}=\frac{a\left(\frac{x_{3}-b}{x_{3}-a}\right)^{\delta_{n-3}}\left(\frac{x_{2}-b}{x_{2}-a}\right)^{\delta_{n-6}}\left(\frac{x_{1}-b}{x_{1}-a}\right)^{\delta_{n-5}}\left(\frac{x_{0}-b}{x_{0}-a}\right)^{\delta_{n-4}-b}}{\left(\frac{x_{3}-b}{x_{3}-a}\right)^{\delta_{n-3}}\left(\frac{x_{2}-b}{x_{2}-a}\right)^{\delta_{n-6}}\left(\frac{x_{1}-b}{x_{1}-a}\right)^{\delta_{n-5}}\left(\frac{x_{0}-b}{x_{0}-a}\right)^{\delta_{n-4}-1}}, \quad n \in \mathbb{N}_{0},
$$

where the sequence $\delta_{n}$ is given by (24).

Remark 1 Equation (7) in the case $k=4, l=2$ is a difference equation with interlacing indices [36], and its general solution is obtained by the general solution to equation (7) in the case $k=2, l=1$. Namely, the subsequences $\left(x_{2 n}\right)_{n \in \mathbb{N}_{0}}$ and $\left(x_{2 n+1}\right)_{n \in \mathbb{N}_{0}}$ are two different solutions of equation (7) in the case $k=2, l=1$. The first solution has the initial values $x_{0}$ and $x_{2}$, whereas the second solution has initial values $x_{1}$ and $x_{3}$. From this we see that these two solutions are not connected to each other.

Case $a=b \neq 0$. Since $a=b \neq 0$, equation (7) becomes

$$
x_{n+k}=\frac{x_{n+l} x_{n}-a^{2}}{x_{n+l}+x_{n}-2 a}, \quad n \in \mathbb{N}_{0} \text {, }
$$

where $k \in \mathbb{N}, l \in \mathbb{N}_{0}, l<k, a \in \mathbb{C} \backslash\{0\}$, and $x_{j} \in \mathbb{C}, j=\overline{0, k-1}$.

In this case equation (13) has only one solution $x^{*}=a$. Hence, it is not possible to use the method in the previous case.

On the other hand, we have

$$
\begin{aligned}
x_{n+k}-a & =\frac{x_{n+l} x_{n}-a\left(x_{n+l}+x_{n}\right)+a^{2}}{x_{n+l}+x_{n}-2 a} \\
& =\frac{\left(x_{n+l}-a\right)\left(x_{n}-a\right)}{x_{n+l}-a+x_{n}-a}
\end{aligned}
$$

for $n \in \mathbb{N}_{0}$.

Equation (26) strikingly suggests a use of the change of variables

$$
y_{n}=x_{n}-a, \quad n \in \mathbb{N}_{0},
$$

by which the equation is transformed to equation (8) where $x_{n}$ is replaced with $y_{n}$. 
From (9) and (27) we see that the change of variables

$$
x_{n}=a+\frac{1}{u_{n}}, \quad n \in \mathbb{N}_{0},
$$

transforms equation (26) to equation (10).

Equation (10) when $\max \{k, l\} \leq 4$ is solvable in a closed form, and the following result holds.

Theorem 3 The following statements hold.

(a) General solution to equation (10) with $k=2$ and $l=1$ is given by the formula

$$
u_{n}=f_{n} u_{1}+f_{n-1} u_{0}, \quad n \in \mathbb{N}_{0},
$$

where $f_{n}$ is the Fibonacci sequence.

(b) General solution to equation (10) with $k=3$ and $l=1$ is given by the formula

$$
u_{n}=\alpha_{n-3} u_{2}+\alpha_{n-2} u_{1}+\alpha_{n-4} u_{0}, \quad n \in \mathbb{N}_{0},
$$

where the sequence $\alpha_{n}$ is given by (21).

(c) General solution to equation (10) with $k=3$ and $l=2$ is given by the formula

$$
u_{n}=\beta_{n-2} u_{2}+\beta_{n-4} u_{1}+\beta_{n-3} u_{0}
$$

where the sequence $\beta_{n}$ is given by (22).

(d) General solution to equation (10) with $k=4$ and $l=1$ is given by the formula

$$
u_{n}=\gamma_{n-5} u_{3}+\gamma_{n-4} u_{2}+\gamma_{n-3} u_{1}+\gamma_{n-6} u_{0},
$$

where the sequence $\gamma_{n}$ is given by (23).

(e) General solution to equation (10) with $k=4$ and $l=2$ is given by the formulas

$$
\begin{aligned}
& u_{2 n}=f_{n} u_{2}+f_{n-1} u_{0}, \quad n \in \mathbb{N}_{0}, \\
& u_{2 n+1}=f_{n} u_{3}+f_{n-1} u_{1}, \quad n \in \mathbb{N}_{0},
\end{aligned}
$$

where $f_{n}$ is the Fibonacci sequence.

(f) General solution to equation (10) with $k=4$ and $l=3$ is given by the formula

$$
u_{n}=\delta_{n-3} u_{3}+\delta_{n-6} u_{2}+\delta_{n-5} u_{1}+\delta_{n-4} u_{0},
$$

where the sequence $\delta_{n}$ is given by (24).

Proof These statements can be easily anticipated and essentially obtained by Theorem 1 . Namely, by using the natural connection (19) between positive solutions to product-type difference equations and some solutions to the corresponding linear difference equations, we see that the above statements follow from the corresponding ones in Theorem 1 for 
the case of such initial values (the solutions to equation (10) with real-valued initial values correspond to some uniquely defined solutions to equation (18) with positive initial values). But since equation (10) is linear, the above formulas are not only its solutions for such initial values, but are obviously solutions for all complex-valued initial values from which all the statements follow.

Remark 2 All the statements in Theorem 3 can be also easily proved by the method of induction. However, the method does not explain the given representations for the solutions to equation (10), unlike the above given proof.

From Theorem 3 and by using relation (28), we obtain the following result.

Theorem 4 Consider equation (7). Assume that $a=b \in \mathbb{C} \backslash\{0\}$. Then the following statements hold.

(a) General solution to equation (7) with $k=2$ and $l=1$ is given by the formula

$$
x_{n}=a+\frac{1}{\frac{f_{n}}{x_{1}-a}+\frac{f_{n-1}}{x_{0}-a}}, \quad n \in \mathbb{N}_{0},
$$

where $f_{n}$ is the Fibonacci sequence.

(b) General solution to equation (7) with $k=3$ and $l=1$ is given by the formula

$$
x_{n}=a+\frac{1}{\frac{\alpha_{n-3}}{x_{2}-a}+\frac{\alpha_{n-2}}{x_{1}-a}+\frac{\alpha_{n-4}}{x_{0}-a}}, \quad n \in \mathbb{N}_{0} \text {, }
$$

where the sequence $\alpha_{n}$ is given by (21).

(c) General solution to equation (7) with $k=3$ and $l=2$ is given by the formula

$$
x_{n}=a+\frac{1}{\frac{\beta_{n-2}}{x_{2}-a}+\frac{\beta_{n-4}}{x_{1}-a}+\frac{\beta_{n-3}}{x_{0}-a}},
$$

where the sequence $\beta_{n}$ is given by (22).

(d) General solution to equation (7) with $k=4$ and $l=1$ is given by the formula

$$
x_{n}=a+\frac{1}{\frac{\gamma_{n-5}}{x_{3}-a}+\frac{\gamma_{n-4}}{x_{2}-a}+\frac{\gamma_{n-3}}{x_{1}-a}+\frac{\gamma_{n-6}}{x_{0}-a}},
$$

where the sequence $\gamma_{n}$ is given by (23).

(e) General solution to equation (7) with $k=4$ and $l=2$ is given by the formula

$$
\begin{aligned}
& x_{2 n}=a+\frac{1}{\frac{f_{n}}{x_{2}-a}+\frac{f_{n-1}}{x_{0}-a}}, \quad n \in \mathbb{N}_{0}, \\
& x_{2 n+1}=a+\frac{1}{\frac{f_{n}}{x_{3}-a}+\frac{f_{n-1}}{x_{1}-a}}, \quad n \in \mathbb{N}_{0},
\end{aligned}
$$

where $f_{n}$ is the Fibonacci sequence. 
(f) General solution to equation (7) with $k=4$ and $l=3$ is given by the formula

$$
x_{n}=a+\frac{1}{\frac{\delta_{n-3}}{x_{3}-a}+\frac{\delta_{n-6}}{x_{2}-a}+\frac{\delta_{n-5}}{x_{1}-a}+\frac{\delta_{n-4}}{x_{0}-a}},
$$

where the sequence $\delta_{n}$ is given by (24).

Remark 3 Note that the previous consideration holds also in the case $a=0$. This means that Theorem 4 gives also general solution to equation (7) in the case $a=b=0$.

Remark 4 For $l=0$, equation (7) becomes a difference equation with interlacing indices and is reduced to the case $l=0$ and $k=1$. In the case $l=0$ and $k=1$, a difference equation of first order is obtained which is solved as explained above. We leave the case to the reader as an exercise.

\section{Acknowledgements}

The work of Zdeněk Šmarda was supported by the project FEKT-S-20-6225 of Brno University of Technology.

Funding

Brno University of Technology, project FEKT-S-20-6225.

Availability of data and materials

Not applicable.

\section{Declarations}

\section{Competing interests}

The authors declare that they have no competing interests.

\section{Authors' contributions}

The authors have contributed equally to the writing of this paper. They read and approved the manuscript.

\section{Author details}

'Mathematical Institute of the Serbian Academy of Sciences, Knez Mihailova 36/III, 11000 Beograd, Serbia. ${ }^{2}$ Department of Medical Research, China Medical University Hospital, China Medical University, Taichung 40402, Taiwan, Republic of China. ${ }^{3}$ Faculty of Electrical Engineering, Belgrade University, Bulevar Kralja Aleksandra 73, 11000 Beograd, Serbia. ${ }^{4}$ Faculty of Mechanical and Civil Engineering in Kraljevo, University of Kragujevac, Kraljevo, Serbia. ${ }^{5}$ Deptartment of Mathematical Sciences, Appalachian State University, Boone, NC 28608, USA. ${ }^{6}$ Department of Mathematics, Faculty of Electrical Engineering and Communication, Brno University of Technology, Technicka 3058/10, CZ - 61600 Brno, Czech Republic.

\section{Publisher's Note}

Springer Nature remains neutral with regard to jurisdictional claims in published maps and institutional affiliations.

Received: 25 August 2021 Accepted: 28 October 2021 Published online: 17 November 2021

\footnotetext{
References

1. Abel, N.H.: Mémoire sur les équations algébriques, ou l'on démontre l'impossibilité de la résolution de l'équation générale du cinquime degré (1824). Abel, N.H., Sylow, L., Lie, S.: Oeuvres Complètes de Niels Henrik Abel, I, 2nd edn., pp. 28-33. Grondahl \& Son (1881) (in French)

2. Bashmakov, M.l., Bekker, B.M., Gol'hovoi, V.M.: Zadachi po Matematike. Algebra i Analiz. Nauka, Moskva (1982) (in Russian)

3. Berg, L., Stević, S.: On some systems of difference equations. Appl. Math. Comput. 218, 1713-1718 (2011)

4. Bernoulli, D:: Observationes de seriebus quae formantur ex additione vel substractione quacunque terminorum se mutuo consequentium, ubi praesertim earundem insignis usus pro inveniendis radicum omnium aequationum algebraicarum ostenditur. Commentarii Acad. Petropol. III 1728, 85-100 (1732) (in Latin)

5. Boole, G.: A Treatsie on the Calculus of Finite Differences, 3rd edn. Macmillan \& Co., London (1880)

6. de Moivre, A.: Miscellanea Analytica de Seriebus et Quadraturis. J. Tonson \& J. Watts, Londini (1730) (in Latin)

7. de Moivre, A.: The Doctrine of Chances, 3rd edn. Strand Publishing, London (1756)

8. Demidovich, B.P., Maron, I.A.: Osnovy Vychislitel'noy Matematiki, 4th edn. Nauka, Moskva (1970) (in Russian)

9. Euler, L.: Introductio in Analysin Infinitorum, Tomus Primus, Lausannae (1748) (in Latin)

10. Fort, T.: Finite Differences and Difference Equations in the Real Domain. Oxford University Press, London (1948)
} 
11. Hildebrand, F.B.: Introduction to Numerical Analysis, 2nd edn. Dover, Mineola (1987)

12. Jordan, C.: Calculus of Finite Differences. Chelsea, New York (1956)

13. Konstaninidis, K., Papaschinopoulos, G., Schinas, C.J.: Asymptotic behaviour of the solutions of systems of partial linear homogeneous and nonhomogeneous difference equations. Math. Methods Appl. Sci. 43(7), 3925-3935 (2020)

14. Krechmar, V.A.: A Problem Book in Algebra. Mir, Moscow (1974)

15. Lagrange, J.-L.: Sur l'intégration d'une équation différentielle à différences finies, qui contient la théorie des suites récurrentes. In: Miscellanea Taurinensia, t. I, pp. 33-42 (1759) (Lagrange OEuvres, I, pp. 23-36, 1867) (in French)

16. Lagrange, J.-L.: OEuvres, t. II. Gauthier-Villars, Paris (1868) (in French)

17. Laplace, P.S.: Recherches sur l'intégration des équations différentielles aux différences finies et sur leur usage dans la théorie des hasards. In: Mémoires de I' Académie Royale des Sciences de Paris 1773, t. VII (1776) (Laplace OEuvres, VIII, pp. 69-197, 1891) (in French)

18. Markoff, A.A.: Differenzenrechnung. Teubner, Leipzig (1896) (in German)

19. Milne-Thomson, L.M.: The Calculus of Finite Differences. Macmillan \& Co., London (1933)

20. Mitrinović, D.S., Kečkić, J.D.: Metodi Izračunavanja Konačnih Zbirova/Methods for Calculating Finite Sums. Naučna Knjiga, Beograd (1984) (in Serbian)

21. Nörlund, N.E.: Vorlesungen über Differenzenrechnung. Springer, Berlin (1924) (in German)

22. Papaschinopoulos, G., Schinas, C.J.: On the behavior of the solutions of a system of two nonlinear difference equations. Commun. Appl. Nonlinear Anal. 5(2), 47-59 (1998)

23. Papaschinopoulos, G., Schinas, C.J.: Invariants for systems of two nonlinear difference equations. Differ. Equ. Dyn. Syst. 7, 181-196 (1999)

24. Papaschinopoulos, G., Schinas, C.J.: Invariants and oscillation for systems of two nonlinear difference equations. Nonlinear Anal., Theory Methods Appl. 46, 967-978 (2001)

25. Papaschinopoulos, G., Stefanidou, G.: Asymptotic behavior of the solutions of a class of rational difference equations. Int. J. Difference Equ. 5(2), 233-249 (2010)

26. Rhouma, M.H.: The Fibonacci sequence modulo $\pi$, chaos and some rational recursive equations. J. Math. Anal. Appl. 310, 506-517 (2005)

27. Schinas, C.: Invariants for difference equations and systems of difference equations of rational form. J. Math. Anal. Appl. 216, 164-179 (1997)

28. Schinas, C.: Invariants for some difference equations. J. Math. Anal. Appl. 212, 281-291 (1997)

29. Stević, S.: General solutions to four classes of nonlinear difference equations and some of their representations. Electron. J. Qual. Theory Differ. Equ. 2019, Article ID 75 (2019)

30. Stević, S.: Solvability of a general class of two-dimensional hyperbolic-cotangent-type systems of difference equations. Adv. Differ. Equ. 2019, Article ID 294 (2019)

31. Stević, S.: Solvability of some classes of nonlinear first-order difference equations by invariants and generalized invariants. Electron. J. Qual. Theory Differ. Equ. 2019, Article ID 36 (2019)

32. Stević, S.: Solving a class of non-autonomous difference equations by generalized invariants. Math. Methods Appl. Sci. 42, 6315-6338 (2019)

33. Stević, S.: New class of practically solvable systems of difference equations of hyperbolic-cotangent-type. Electron. J. Qual. Theory Differ. Equ. 2020, Article ID 89 (2020)

34. Stević, S.: On a class of solvable difference equations generalizing an iteration process for calculating reciprocals. Adv. Differ. Equ. 2021, Article ID 205 (2021)

35. Stević, S., Iričanin, B., Kosmala, W.: More on a hyperbolic-cotangent class of difference equations. Math. Methods Appl. Sci. 42, 2974-2992 (2019)

36. Stević, S., Iričanin, B., Kosmala, W., Šmarda, Z.: Note on the bilinear difference equation with a delay. Math. Methods Appl. Sci. 41, 9349-9360 (2018)

37. Stević, S., Iričanin, B., Šmarda, Z.: Solvability of a close to symmetric system of difference equations. Electron. J. Differ. Equ. 2016, Article ID 159 (2016)

38. Vorobiev, N.N.: Fibonacci Numbers. Birkhäuser, Basel (2002)

\section{Submit your manuscript to a SpringerOpen ${ }^{\circ}$ journal and benefit from:}

- Convenient online submission

- Rigorous peer review

- Open access: articles freely available online

- High visibility within the field

- Retaining the copyright to your article

Submit your next manuscript at $\gg$ springeropen.com 